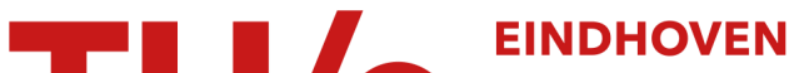 UNIVERSITY OF TECHNOLOGY
}

\section{A Bayesian MMSE turbo receiver for coded MIMO systems}

\section{Citation for published version (APA):}

Sun, S., Wu, Y., \& Tjhung, T. T. (2008). A Bayesian MMSE turbo receiver for coded MIMO systems. IEEE

Transactions on Vehicular Technology, 57(5), 3283-3293. https://doi.org/10.1109/TVT.2008.917230

DOI:

10.1109/TVT.2008.917230

Document status and date:

Published: 01/01/2008

\section{Document Version:}

Publisher's PDF, also known as Version of Record (includes final page, issue and volume numbers)

\section{Please check the document version of this publication:}

- A submitted manuscript is the version of the article upon submission and before peer-review. There can be important differences between the submitted version and the official published version of record. People interested in the research are advised to contact the author for the final version of the publication, or visit the $\mathrm{DOI}$ to the publisher's website.

- The final author version and the galley proof are versions of the publication after peer review.

- The final published version features the final layout of the paper including the volume, issue and page numbers.

Link to publication

\section{General rights}

Copyright and moral rights for the publications made accessible in the public portal are retained by the authors and/or other copyright owners and it is a condition of accessing publications that users recognise and abide by the legal requirements associated with these rights.

- Users may download and print one copy of any publication from the public portal for the purpose of private study or research.

- You may not further distribute the material or use it for any profit-making activity or commercial gain

- You may freely distribute the URL identifying the publication in the public portal.

If the publication is distributed under the terms of Article 25fa of the Dutch Copyright Act, indicated by the "Taverne" license above, please follow below link for the End User Agreement:

www.tue.nl/taverne

Take down policy

If you believe that this document breaches copyright please contact us at:

openaccess@tue.nl

providing details and we will investigate your claim. 


\section{A Bayesian MMSE Turbo Receiver for Coded MIMO Systems}

\author{
Sumei Sun, Yan Wu, Yuan Li, and Tjeng Thiang Tjhung
}

\begin{abstract}
In conventional interference cancellation (IC)-based turbo receivers, extrinsic information (EXT) from the soft-input-soft-output (SISO) decoders is used to compute the statistical mean (SM) of the interfering signals. In this paper, we present a class of Bayesian minimum mean-square-error (MMSE) turbo receivers for coded multiple-inputmultiple-output (MIMO) systems. Instead of using the EXT to estimate the prior SM, we use it in the posterior Bayesian MMSE estimation of the interfering signals. The estimation accuracy is, therefore, improved, leading to better bit-error-rate (BER) performance from our proposed Bayesian receivers. For the cases that we have studied, the proposed receiver with $t w o$ iterations has a lower BER than the conventional one with five iterations, thus significantly reducing the receiver processing delay and the implementation complexity. Its superior performance is also demonstrated by the higher detector output mutual information and fewer iterations to achieve convergence, which is obtained from the extrinsic information transfer (EXIT) chart analysis.
\end{abstract}

Index Terms-Bayesian estimation (BE), expectation maximization (EM), extrinsic information transfer (EXIT) chart, multiple-inputmultiple-output (MIMO), turbo receiver.

\section{INTRODUCTION}

Multiple-input-multiple-output (MIMO) wireless communication systems have attracted a lot of academic and industrial interest over the last decade due to their great potential to increase spectral efficiency and diversity gains. Many schemes have been proposed to realize these gains, for example, the various Bell Laboratories Layered Space-Time (BLAST) architectures [1], [2] to exploit multiplexing gain, space-time block codes (STBCs) [3], [4] to exploit diversity gain, and the simple hybrid schemes of STBCs and spatial multiplexing such as groupwise STBCs (GSTBCs) [5] to exploit multiplexing and diversity gains.

In this paper, we focus on iterative turbo receiver techniques for forward error control-coded MIMO systems. In this context, many works have been published. For example, Lu and Wang [6] proposed a linear minimum mean-square-error (LMMSE) interference cancellation (IC) based turbo receiver for multiuser space-time coded systems; Sellathurai and Haykin [7] applied the same receiver to coded BLAST systems and referred to it as the turbo BLAST (T-BLAST). The biterror-rate (BER) performance of the T-BLAST in correlated Rayleigh fading channels was then evaluated in [8], and its practical virtue was verified in experiments in [9]. In a semitutorial paper, Biglieri et al. [10] analyzed the performance of the IC-based turbo receivers using the extrinsic information transfer (EXIT) chart. Boutros and Caire [11] developed a more generalized framework on iterative receivers for code-division multiple-access (CDMA) systems, which is also applicable to MIMO. Based on the factor-graph representation and the sumproduct algorithm [12], they showed that the estimated interference at each iteration should be a function of the decoders' extrinsic information (EXT) rather than the a posteriori probability. The EXT

Manuscript received June 2, 2006; revised November 5, 2006, April 30, 2007, October 24, 2007, and December 3, 2007. The review of this paper was coordinated by Dr. A. Ghrayeb.

The authors are with the Institute for Infocomm Research, Singapore 119613 (e-mail: sunsm@i2r.a-star.edu.sg; wuyan@i2r.a-star.edu.sg; liyuan@i2r.a-star. edu.sg; tjhungtt@i2r.a-star.edu.sg).

Color versions of one or more of the figures in this paper are available online at http://ieeexplore.ieee.org.

Digital Object Identifier 10.1109/TVT.2008.917230 is used to obtain the a priori probability of the coded bits from which the statistical mean (SM), or the prior estimate [13], of the interference is calculated. IC is then performed, and soft-input-softoutput (SISO) decoding is implemented. Different filtering schemes, such as a matched filter (MF) or the LMMSE, can be applied in the IC step. In MIMO, we interchangeably use the terms maximal ratio combining (MRC) and MF. The turbo receivers considered in [6]-[11] are referred to as conventional turbo receivers in this paper.

One drawback to the conventional schemes, as we will show in this paper, lies in the poor accuracy of the SM estimate at low-to-medium SNR values or when a high rate code is used. To improve receiver performance, we propose a novel Bayesian minimum mean-squareerror (BMMSE) turbo receiver that exploits the EXT in the Bayesian estimation (BE) [13, ch. 10 and 11], [14, Lesson 13] of the interference signals. We show that the $\mathrm{BE}$ conditioned on the received signal and the estimated interference (from the previous iteration) is a function of both the EXT and the decision statistic of the IC-MRC. The iterative $\mathrm{IC}$ receiver based on $\mathrm{BE}$ is referred to as the Bayesian IC-MRC turbo receiver in this paper to differentiate it from the conventional design in [11]. We show through EXIT chart analysis and BER simulations that the posterior BE results in much higher output mutual information at the SISO detector, fewer iterations to achieve convergence to the lower bound, and a lower BER than the conventional prior SM estimate.

Using the Gaussian model that is developed in [15] for the conventional LMMSE-IC detectors, we further apply the BE scheme to LMMSE-IC receivers and develop a class of Bayesian LMMSE-IC turbo receivers. It can have accurate interference estimation by $\mathrm{BE}$ and effective interference suppression by LMMSE filtering; therefore, it has superior performance over the conventional LMMSE-IC receivers. This will be demonstrated in the EXIT chart analysis and the BER simulation results.

The application of Bayesian expectation maximization (EM)maximum a posteriori (MAP) symbol estimation in a turbo receiver was previously considered in [16], in which the frequency flat-fading channel coefficients and the transmitted symbols were estimated iteratively. However, no detailed derivation and analysis were given in [16].

In this paper, we consider both narrow-band signals in flat-fading channels and wideband signals in frequency-selective channels. In the latter case, the multipath-induced intersymbol interference (ISI) is mitigated by orthogonal frequency division multiplexing (OFDM) [17]. MIMO-OFDM is of high practical interest, which has been selected as the enabling technology for the next-generation high-throughput wireless local area networks (WLANs) in IEEE 802.11n [18]. We will establish a common signal model for the frequency-domain (FD) signals in MIMO-OFDM and the time-domain signals in narrow-band MIMO systems. For the applications, we consider the vertical BLAST (VBLAST) and GSTBC OFDM systems, both of which have been adopted in IEEE 802.11n [18]. The proposed receiver design, however, can be applied to multiuser CDMA systems in a straightforward manner.

Throughout this paper, vectors and matrices are represented by boldfaced letters. The symbols $(\cdot)^{T},(\cdot)^{H}$, and $(\cdot)^{-1}$ denote matrix transposition, Hermitian, and inversion, respectively. All vectors are defined as column vectors, with row vectors represented by transposition. We use $\breve{X}, \hat{X}$, and $\tilde{X}$ to, respectively, denote the BMMSE estimate, the SM estimate, and the detector decision statistic for the signal $X$.

\section{Signal Model}

We consider the wideband signals in frequency-selective channels and the narrow-band signals in flat-fading channels. For the former 


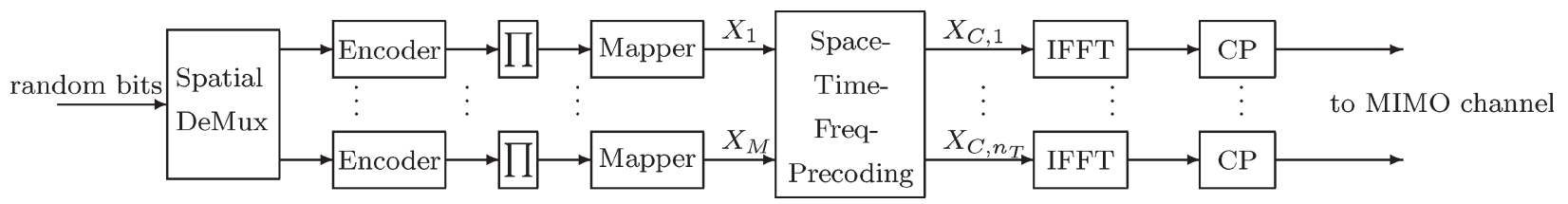

Fig. 1. Coded MIMO-OFDM transmitter. $\prod$ denotes the bit interleaver.

case, the multipath induced ISI can be mitigated by OFDM modulation. The coded MIMO-OFDM system that is considered in this paper is depicted in Fig. 1. The random information bits are first demultiplexed into parallel streams (layers) by the "Spatial DeMux" unit. In each parallel stream, information bits are encoded, bit-interleaved, and mapped to constellation points of the adopted modulation scheme based on the bit-interleaved coded modulation (BICM) principle [19]. This is referred to as a "horizontal coding architecture" (HCA) [20]. Alternately, a "vertical coding architecture" (VCA) [20] can be adopted. In the VCA, a single encoder is used. The encoded bits are demultiplexed into parallel streams, and the bit-interleaving and symbol-mapping functions are implemented in each stream. Both coding architectures have been adopted in IEEE 802.11n [18]. The signal model that is established in this section and the BMMSE turbo receivers that are proposed in this paper can be applied to both architectures.

The BICM outputs from the parallel streams are further processed by the "space-time-frequency precoding" (STFP) unit. Depending on the system requirement and the available transmit and receive antennas, different STFP schemes can be adopted. For example, if maximum multiplexing gain is desired and if no channel state information (CSI) is available at the transmitter, VBLAST can be used. If both transmit diversity and multiplexing gains are desired, GSTBCs [5] can be applied.

The STFP output is then OFDM-modulated, consisting of the inverse fast Fourier transform and cyclic prefix $(\mathrm{CP})$ insertion. The $\mathrm{CP}$ length should be no shorter than the number of multipaths in the channel to completely remove the ISI [17]. The OFDM-modulated signals are then transmitted by different antennas.

Assuming perfect timing and frequency synchronization, we can remove the $\mathrm{CP}$ part from the received data before converting them to the FD by fast Fourier transform (FFT), as shown in Fig. 2. The FD data at each receive antenna are then multiplexed into one signal vector $\mathbf{R}$ for each subcarrier, which is written as follows:

$$
\mathbf{R}=\mathbf{H X}+\mathbf{V}
$$

where $\mathbf{H}, \mathbf{X}$, and $\mathbf{V}$ denote the channel, the transmitted signal, and the additive white Gaussian noise (AWGN), respectively. For a VBLAST system with $n_{\mathrm{T}}$ transmit and $n_{\mathrm{R}}$ receive antennas $\left(n_{\mathrm{T}} \times n_{\mathrm{R}}\right)$, we have

$$
\begin{aligned}
& \mathbf{R} \stackrel{\text { def }}{=}\left[\begin{array}{llll}
R_{1} & R_{2} & \cdots & R_{n_{\mathrm{R}}}
\end{array}\right]_{n_{\mathrm{R}} \times 1}^{T} \\
& \mathbf{H} \stackrel{\text { def }}{=}\left[H_{i, j}\right]_{n_{\mathrm{R}} \times n_{\mathrm{T}}} \\
& \mathbf{X} \stackrel{\text { def }}{=}\left[\begin{array}{llll}
X_{1} & X_{2} & \cdots & X_{n_{\mathrm{T}}}
\end{array}\right]_{n_{\mathrm{T}} \times 1}^{T} \\
& \mathbf{V} \stackrel{\text { def }}{=}\left[\begin{array}{llll}
V_{1} & V_{2} & \cdots & V_{n_{\mathrm{R}}}
\end{array}\right]_{n_{\mathrm{R}} \times 1}^{T}
\end{aligned}
$$

where the subscripts for the elements in $\mathbf{R}$ and $\mathbf{V}$ denote the receive antenna index, the subscript in $\mathbf{X}$ denotes the transmit antenna index, and the subscript in $\mathbf{H}$ denotes the receive-transmit antenna pair index. For the complex AWGN, we have $\mathbf{V} \sim C N\left(0,2 \sigma^{2} \mathbf{I}_{n_{\mathrm{R}}}\right)$.
For an $n_{\mathrm{T}} \times n_{\mathrm{R}}$ GSTBC system, we have

$$
\begin{aligned}
& \mathbf{R} \stackrel{\text { def }}{=}\left[\begin{array}{lllll}
R_{1,1} & -R_{1,2}^{*} & \cdots & R_{n_{\mathrm{R}}, 1} & -R_{n_{\mathrm{R}}, 2}^{*}
\end{array}\right]_{2 n_{\mathrm{R}} \times 1}^{T} \\
& \mathbf{X} \stackrel{\text { def }}{=}\left[\begin{array}{lllll}
X_{1} & X_{2} & \cdots & X_{n_{\mathrm{T}}}
\end{array}\right]_{n_{\mathrm{T}} \times 1}^{T} \\
& \mathbf{V} \stackrel{\text { def }}{=}\left[\begin{array}{lllll}
V_{1,1} & -V_{1,2}^{*} & \cdots & V_{n_{\mathrm{R}}, 1} & -V_{n_{\mathrm{R}}, 2}^{*}
\end{array}\right]_{n_{\mathrm{R}} \times 1}^{T} \\
& \mathbf{H} \stackrel{\text { def }}{=}\left[\begin{array}{lll}
\left(\mathbf{H}_{i, j}\right)_{2 \times 2} & ]_{2 n_{\mathrm{R}} \times n_{\mathrm{T}}}
\end{array}\right.
\end{aligned}
$$

where

$$
\left(\mathbf{H}_{i, j}\right)_{2 \times 2}=\left[\begin{array}{cc}
H_{i, 2 j-1} & H_{i, 2 j} \\
-H_{i, 2 j}^{*} & H_{i, 2 j-1}^{*}
\end{array}\right], \quad i=1, \ldots, n_{\mathrm{R}}, j=1, \ldots, \frac{n_{\mathrm{T}}}{2}
$$

and the subscripts of $R$ and $V$ denote the receive antenna and OFDM symbol indexes in each Alamouti STBC codeword [5].

The signal model in (1) is applicable to single-carrier systems in narrow-band, flat-fading channels. In this case, no OFDM modulation is needed, and the STFP block is replaced by a space-time precoding block. Accordingly, (1) becomes a time-domain signal model. Other than that, the rest of the blocks remain the same as for the MIMOOFDM systems.

\section{BAYESIAN IC-MRC TURBo RECEIVER}

The proposed Bayesian turbo receiver for MIMO-OFDM systems with an HCA is depicted in Fig. 2. When we remove the OFDM demodulation blocks, the same receiver can be directly applied to narrow-band, single-carrier systems. Besides the OFDM demodulation and spatial multiplexing units, the rest of the receiver can be divided into two parts- the SISO detector and the SISO decoders. The EXT is exchanged between the two parts, as we will show in detail here and in Section IV. At each iteration, the SISO decoders take in the deinterleaved extrinsic bit metric values from the SISO detector and compute the updated extrinsic log-likelihood ratio (LLR), i.e., the EXT, for the coded bits. The updated EXT is then interleaved and sent back to the SISO detector.

The SISO detector consists of three blocks-the BMMSE estimators, the STFP detector, and the soft demodulator. In the BMMSE estimators, the BE of the transmitted signals is calculated using the EXT of the SISO decoder and the decision statistic of the STFP detector, both from the previous iteration. Their outputs are used by the STFP detector to implement either the IC-MRC or the LMMSE-IC scheme. The detector output, i.e., the decision statistic of the transmitted signals, is then delivered to the soft demodulator to calculate the extrinsic bit metric values.

\section{A. STFP Detector}

The STFP detector calculates the decision statistic of the transmitted signals. For the low-complexity, IC-based linear detectors, the decision statistic is calculated as

$$
\tilde{X}_{k, i}=\mathbf{F}_{k, i}^{H} \tilde{\mathbf{R}}_{k, i}
$$




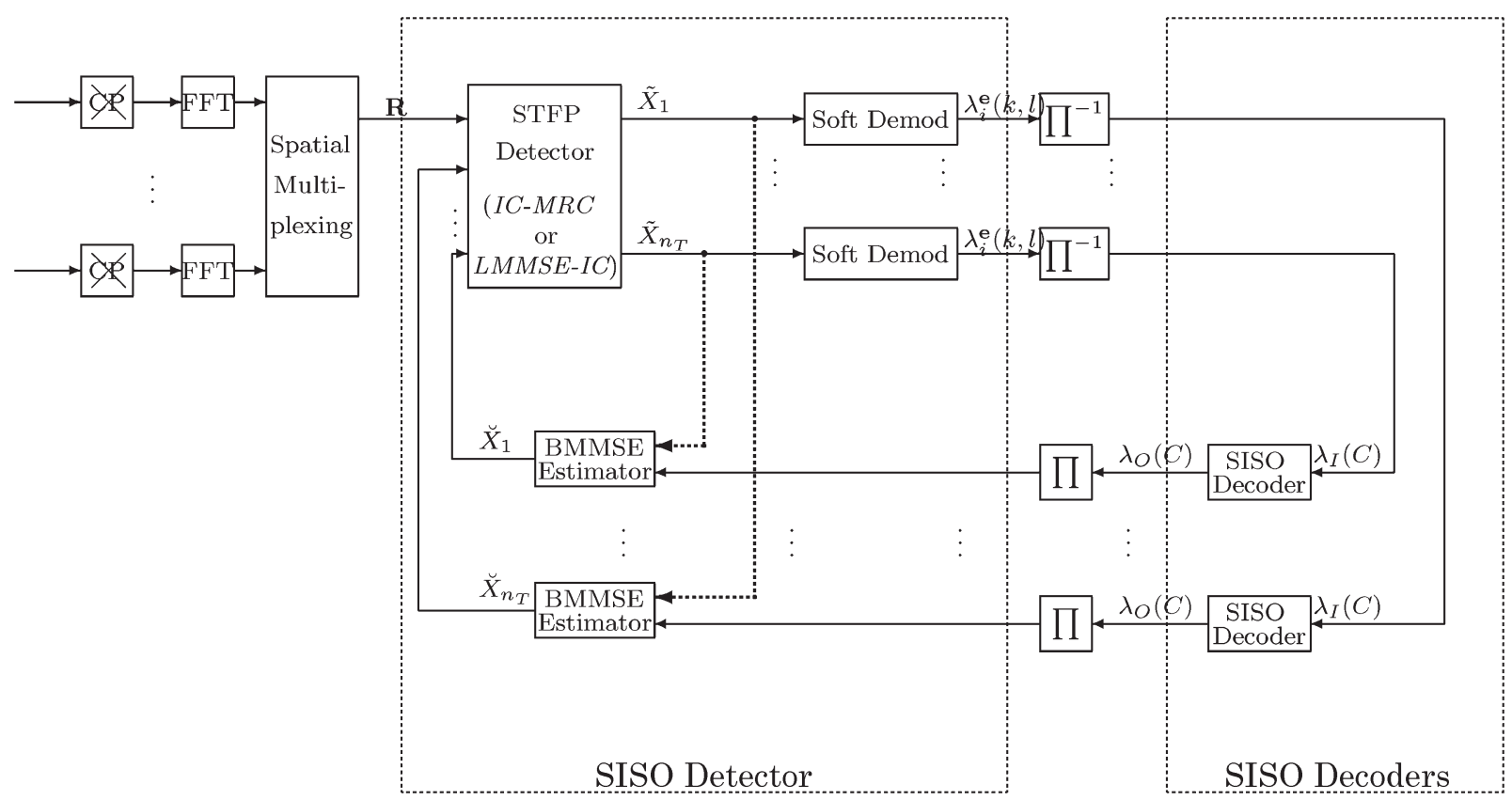

Fig. 2. Bayesian turbo receiver.

where the subscripts $k$ and $i$ represent the signal and iteration indexes, respectively, and $\tilde{\mathbf{R}}$ denotes the IC output, which is given as

$$
\tilde{\mathbf{R}}_{k, i}=\mathbf{R}-\sum_{\substack{p=1 \\ p \neq k}}^{n_{\mathrm{T}}} \mathbf{H}_{p} X_{p, i-1, \mathrm{est}}=\mathbf{H}_{k} X_{k}+\tilde{\mathbf{V}}_{k, i}
$$

with $\mathbf{H}_{p}$ denoting the $p$ th column of $\mathbf{H}, X_{p, i-1 \text {,est }}$ denoting the estimate of $X_{p}$ at iteration $(i-1)$, and $\tilde{\mathbf{V}}_{k, i}$ denoting the composite residual interference and AWGN, i.e.,

$$
\tilde{\mathbf{V}}_{k, i}=\sum_{\substack{p=1 \\ p \neq k}}^{n_{\mathrm{T}}} \mathbf{H}_{p}\left(X_{p}-X_{p, i-1, \mathrm{est}}\right)+\mathbf{V}
$$

F denotes the linear filter for which two schemes can be implemented, namely, an MF (or MRC) with

$$
\mathbf{F}_{k, i}=\mathbf{H}_{k}
$$

and the LMMSE filter to be discussed in detail in Section IV.

In conventional turbo receivers, the $\mathrm{SM}$ is used for $X_{p, i-1, \text { est }}$, which is computed as follows [10], [11]:

$$
\hat{X}_{p, i-1}=\sum_{X_{m} \in \Omega} X_{m} p_{i-1}\left(X_{m}\right)
$$

where $\Omega$ is the modulation set of the transmitted signals with $\operatorname{dim}(\Omega)=M$, and $p_{i-1}\left(X_{m}\right)$ is the probability mass function (pmf) that is calculated at iteration $(i-1)$, i.e.,

$$
p_{i-1}\left(X_{m}\right)=\prod_{l=1}^{\log _{2} M} p_{i-1}\left(c_{m}^{l}\right)
$$

under the assumption that $X_{m}$ is mapped from bits $\left[c_{m}^{1}, c_{m}^{2}, \ldots\right.$, $c_{m}^{\log _{2} M}$, which are uncorrelated with sufficient interleaving. $p_{i-1}\left(c_{m}^{l}\right)$ denotes the a priori pmf estimate for bit $c_{m}^{l}$ [6], i.e.,

$$
p_{i-1}\left(c_{k}^{l}\right)=\exp \left(\frac{\lambda_{i-1}^{\mathrm{a}}(k, l) c_{k}^{l}}{1+\exp \left(\lambda_{i-1}^{\mathrm{a}}(k, l)\right)}\right)
$$

with $\lambda_{i-1}^{\mathrm{a}}(k, l)$ being the interleaved EXT for $c_{k}^{l}$ at iteration $(i-1)$ and the superscript "a" denoting a priori. For notational simplicity, we will omit the iteration index in the pmf for the rest of this paper.

In this paper, we take a different detection approach. We start from the optimal BMMSE estimate of the transmitted signals and show that when EM algorithm [21] is used to reduce the computational complexity, we obtain the same linear IC-MRC detector in (2) and (4). However, in the IC process [see (3)], the BMMSE estimates $\left\{\breve{X}_{p, i-1}\right\}$, instead of the prior SM estimate $\left\{\hat{X}_{p, i-1}\right\}$, of the interference signals will be used. This is one major difference of our proposed BMMSE turbo receiver from its conventional counterpart.

\section{B. Optimal BMMSE Estimate}

Assuming perfect CSI at the receiver, we obtain the BMMSE estimate of the transmitted signals as [13, ch. 10 and 11]

$$
\begin{aligned}
\breve{\mathbf{X}}_{\mathrm{BMMSE}} & =E\{\mathbf{X} \mid \mathbf{R}\} \\
& =\sum_{\mathbf{X}_{j} \in \Omega^{n_{\mathrm{T}}}} \mathbf{X}_{j} p\left(\mathbf{X}_{j} \mid \mathbf{R}\right) \\
& =\frac{\sum_{\mathbf{x}_{j} \in \Omega^{n_{\mathrm{T}}}} \mathbf{X}_{j} p\left(\mathbf{R} \mid \mathbf{X}_{j}\right) p\left(\mathbf{X}_{j}\right)}{\sum_{\mathbf{X}_{i} \in \Omega^{n} \mathrm{~T}} p\left(\mathbf{R} \mid \mathbf{X}_{i}\right) p\left(\mathbf{X}_{i}\right)}
\end{aligned}
$$

where the last equality is obtained by using Bayes' rule.

The computation of the numerator and the denominator of (8) incurs the complexity of $M^{n_{\mathrm{T}}}$. Simplification is, thus, desired. To reduce the computational complexity, we adopt the expectation step of the spacealternating missing parameter EM algorithm that is outlined in [21]. In this approach, the transmitted signal is estimated by treating the interfering signals as "missing" data. 


\section{C. $B E M E$}

The Bayesian EM MMSE estimate (BEME) of $X_{k}$ at iteration $i$, i.e., $\breve{X}_{k, i}$, is derived based on the received signal $\mathbf{R}$ and the BEME of the interfering signals at iteration $i-1$, i.e., $\breve{X}_{p, i-1}, p \neq k$, i.e.,

$$
\breve{X}_{k, i} \stackrel{\text { def }}{=} E\left\{X_{k} \mid \mathbf{R}, \breve{\mathbf{X}}_{k, i-1}\right\}
$$

where $\breve{\mathbf{X}}_{k, i-1}=\left[\breve{X}_{1, i-1}, \ldots, \breve{X}_{k-1, i-1}, \breve{X}_{k+1, i-1}, \ldots, \breve{X}_{n_{\mathrm{T}}, i-1}\right]^{T}$.

Using Bayes' rule, we can further write (9) as follows:

$$
\begin{aligned}
\breve{X}_{k, i} & =\sum_{S_{m} \in \Omega} S_{m} p\left(X_{k}=S_{m} \mid \mathbf{R}, \overline{\mathbf{X}}_{k, i-1}\right) \\
& =\sum_{S_{m} \in \Omega} S_{m} \frac{p\left(X_{k}=S_{m}, \mathbf{R}, \overline{\mathbf{X}}_{k, i-1}\right)}{p\left(\mathbf{R}, \overline{\mathbf{X}}_{k, i-1}\right)} \\
& =\sum_{S_{m} \in \Omega} S_{m} \frac{p\left(\mathbf{R} \mid X_{k}=S_{m}, \overline{\mathbf{X}}_{k, i-1}\right) p\left(X_{k}=S_{m}, \overline{\mathbf{X}}_{k, i-1}\right)}{\sum_{S_{n} \in \Omega} p\left(\mathbf{R}, X_{k}=S_{n}, \breve{\mathbf{X}}_{k, i-1}\right)} \\
& =\frac{\sum_{m} \in \Omega}{\sum_{S_{n} \in \Omega} p\left(\mathbf{R} \mid X_{k}=S_{n}, \breve{\mathbf{X}}_{k, i-1}\right) p\left(X_{k}=S_{n}\right)}
\end{aligned}
$$

under the assumption that $p\left(X_{k}, \overline{\mathbf{X}}_{k, i-1}\right)=p\left(X_{k}\right) p\left(\overline{\mathbf{X}}_{k, i-1}\right)$.

The conventional EM receivers in [21] and [22] assume an equal pmf for the transmitted signals; hence, (10) becomes

$$
\breve{X}_{k, i}=\frac{\sum_{S_{m} \in \Omega} S_{m} p\left(\mathbf{R} \mid X_{k}=S_{m}, \overline{\mathbf{X}}_{k, i-1}\right)}{\sum_{S_{n} \in \Omega} p\left(\mathbf{R} \mid X_{k}=S_{n}, \overline{\mathbf{X}}_{k, i-1}\right)} .
$$

In this paper, we propose to use the estimated pmf in (6) in (10). This distinguishes our approach from the conventional one in (11). In terms of the application of the turbo principle, using the a priori information in the posterior BEME is different from using it in the prior SM estimation in the conventional turbo receivers.

Theorem 1: The BEME $\breve{X}_{k, i}$ is unbiased.

Proof: From (9), we have

$$
\begin{aligned}
E\left\{\breve{X}_{k, i}\right\} & =E\left\{E\left\{X_{k} \mid \mathbf{R}, \breve{\mathbf{X}}_{k, i-1}\right\}\right\} \\
& =E\left\{\sum_{X_{k} \in \Omega} X_{k} p\left(X_{k} \mid \mathbf{R}, \overline{\mathbf{X}}_{k, i-1}\right)\right\} \\
& =\int p\left(\mathbf{R} \mid \overline{\mathbf{X}}_{k, i-1}\right) d \mathbf{R} \sum_{X_{k} \in \Omega} X_{k} p\left(X_{k} \mid \mathbf{R}, \overline{\mathbf{X}}_{k, i-1}\right) \\
& =\sum_{X_{k} \in \Omega} X_{k} \int p\left(\mathbf{R} \mid \overline{\mathbf{X}}_{k, i-1}\right) p\left(X_{k} \mid \mathbf{R}, \overline{\mathbf{X}}_{k, i-1}\right) d \mathbf{R} \\
& =\sum_{X_{k} \in \Omega} X_{k} \int p\left(X_{k}, \mathbf{R} \mid \breve{\mathbf{X}}_{k, i-1}\right) d \mathbf{R} \\
& =\sum_{X_{k} \in \Omega} X_{k} p\left(X_{k} \mid \overline{\mathbf{X}}_{k, i-1}\right) \\
& =\sum_{X_{k} \in \Omega} X_{k} p\left(X_{k}\right)=E\left\{X_{k}\right\} .
\end{aligned}
$$

For the modulation schemes that are considered here, we further have

$$
E\left\{\breve{X}_{k, i}\right\}=0 .
$$

Now, we proceed to derive BEME $\breve{X}_{k, i}$. We first assume perfect interference estimation and cancellation. In this case, we have

$$
p\left(\mathbf{R} \mid X_{k}, \breve{\mathbf{X}}_{k, i-1}\right)=\alpha \exp \left[-\frac{(\mathbf{R}-\mathrm{HX})^{H}(\mathbf{R}-\mathrm{HX})}{2 \sigma^{2}}\right]
$$

with $\mathbf{X}=\left[X_{k}, \breve{\mathrm{X}}_{k, i-1}\right]$, and $\alpha$ is a system-dependent constant, e.g., $\alpha=1 /\left(2 \pi \sigma^{2}\right)^{n_{\mathrm{R}}}$ for the VBLAST, and $\alpha=1 /\left(2 \pi \sigma^{2}\right)^{2 n_{\mathrm{R}}}$ for the GSTBC

To simplify the derivation, we define

$$
\begin{aligned}
\kappa & =\left.(\mathbf{R}-\mathbf{H X})^{H}(\mathbf{R}-\mathbf{H} \mathbf{X})\right|_{\mathbf{X}=\left[X_{k}, \breve{\mathbf{X}}_{k, i-1}\right]} \\
& =\mathbf{R}^{H} \mathbf{R}-2 \operatorname{Re}\left(\mathbf{X}^{H} \mathbf{H}^{H} \mathbf{R}\right)+\mathbf{X}^{H} \mathbf{H}^{H} \mathbf{H} \mathbf{X} \\
& =\varrho+\left\|\mathbf{H}_{k} X_{k}\right\|^{2}-2 \operatorname{Re}\left(X_{k}^{*} \mathbf{H}_{k}^{H} \mathbf{R}-\sum_{\substack{p=1 \\
p \neq k}}^{n_{T}} H_{k}^{H} \mathbf{H}_{p} X_{k}^{*} \breve{X}_{p, i-1}\right) \\
& =\varrho+\left\|\mathbf{H}_{k} X_{\mathbf{k}}\right\|^{2}-2 \operatorname{Re}\left[X_{k}^{*} \mathbf{H}_{k}^{H}\left(\mathbf{R}-\sum_{\substack{p=1 \\
p \neq k}}^{n_{T}} \mathbf{H}_{p} \breve{X}_{p, i-1}\right)\right] \\
& =\varrho+\left\|\mathbf{H}_{k} X_{k}\right\|^{2}-2 \operatorname{Re}\left(X_{k}^{*} \tilde{X}_{k, i}\right)
\end{aligned}
$$

where "Re" denotes the real part of the signal

$$
\begin{aligned}
\varrho=\mathbf{R}^{H} \mathbf{R}-2 \operatorname{Re}\left(\sum_{\substack{p=1 \\
p \neq k}}^{n_{\mathrm{T}}} \breve{X}_{p, i-1}^{*} \mathbf{H}_{p}^{H} \mathbf{R}\right) \\
+\sum_{\substack{p=1 \\
p \neq k}}^{n_{\mathrm{T}}} \sum_{\substack{q=1 \\
q \neq k}}^{n_{\mathrm{T}}} \mathbf{H}_{p}^{H} \mathbf{H}_{q} \breve{X}_{p, i-1}^{*} \breve{X}_{q, i-1}
\end{aligned}
$$

is a constant term for all the hypotheses of $X_{k}$, and

$$
\tilde{X}_{k, i}=\mathbf{H}_{k}^{H}\left(\mathbf{R}-\sum_{\substack{p=1 \\ p \neq k}}^{n_{\mathrm{T}}} \mathbf{H}_{p} \breve{X}_{p, i-1}\right)
$$

is the IC-MRC decision statistic in (2).

Equation (12) is, therefore, simplified to

$$
p\left(\mathbf{R} \mid X_{k}, \overline{\mathbf{X}}_{k, i-1}\right)=\mathcal{C} \exp \left[-\frac{\left\|\mathbf{H}_{k} X_{k}\right\|^{2}-2 \operatorname{Re}\left(X_{k}^{*} \tilde{X}_{k, i}\right)}{2 \sigma^{2}}\right]
$$

where $\mathcal{C}=\alpha \exp \left(-\varrho / 2 \sigma^{2}\right)$, and the BEME in (10) becomes

$$
\breve{X}_{k, i}=\frac{\sum_{S_{m} \in \Omega} S_{m} \exp \left[\frac{-\left\|\mathbf{H}_{k} S_{m}\right\|^{2}+2 \operatorname{Re}\left(S_{m}^{*} \tilde{X}_{k, i}\right)}{2 \sigma^{2}}\right] p\left(X_{k}=S_{m}\right)}{\sum_{S_{n} \in \Omega} \exp \left[\frac{-\left\|\mathbf{H}_{k} S_{n}\right\|^{2}+2 \operatorname{Re}\left(S_{n}^{*} \tilde{X}_{k, i}\right)}{2 \sigma^{2}}\right] p\left(X_{k}=S_{n}\right)} .
$$

From (14), we can see that to obtain $\breve{X}_{k, i}$, we need $\tilde{X}_{k, i}$ and $p\left(X_{k}\right)$. Whereas $p\left(X_{k}\right)$ can be obtained from the SISO decoders, as shown in Fig. 2, an IC-MRC detector needs to be implemented to provide $\tilde{X}_{k, i}$. 
For constant modulus modulation signals, (14) can be further simplified to

$$
\breve{X}_{k, i}=\frac{\sum_{S_{m} \in \Omega} S_{m} \exp \left[\operatorname{Re}\left(\frac{S_{m}^{*} \tilde{X}_{k, i}}{\sigma^{2}}\right)\right] p\left(X_{k}=S_{m}\right)}{\sum_{S_{n} \in \Omega} \exp \left[\operatorname{Re}\left(\frac{S_{n}^{*} \tilde{X}_{k, i}}{\sigma^{2}}\right)\right] p\left(X_{k}=S_{n}\right) .}
$$

The assumption of perfect IC, however, is unrealistic. On the other hand, obtaining an accurate estimate of the multivariate conditional pdf $p\left(\mathbf{R} \mid X_{k}, \breve{\mathbf{X}}_{k, i-1}\right)$ is very complex. As an alternative, we proceed to analyze the statistical properties of the decision statistic of the ICMRC $\tilde{X}_{k, i}$ and obtain its conditional pdf $p\left(\tilde{X}_{k, i} \mid X_{k}\right)$, as suggested by (13) and (14). We will then obtain the BEME by using $\tilde{X}_{k, i}$ as the observation signal.

1) Statistics of the IC-MRC Signal: From (2) and (4), we can express the decision statistic of IC-MRC as

$$
\tilde{X}_{k, i}=g_{k} X_{k}+\tilde{v}_{k, i}
$$

where

$$
\begin{aligned}
g_{k} & =\mathbf{H}_{k}^{H} \mathbf{H}_{k} \\
\tilde{v}_{k, i} & =\mathbf{H}_{k}^{H} \tilde{\mathbf{V}}_{k, i}=\sum_{\substack{p=1 \\
p \neq k}}^{n_{\mathrm{T}}} \mathbf{H}_{k}^{H} \mathbf{H}_{p}\left(X_{p}-\breve{X}_{p, i-1}\right)+\mathbf{H}_{k}^{H} \mathbf{V} .
\end{aligned}
$$

When $n_{\mathrm{T}}$ is reasonably large, $\tilde{v}_{k, i}$ can be approximated as a Gaussian random variable from the central limit theorem. As proven in Appendix A, it has mean zero and variance

$$
\varsigma^{2}=\frac{1}{2} \sum_{\substack{p=1 \\ p \neq k}}^{n_{\mathrm{T}}} \frac{n_{\mathrm{R}}}{n_{\mathrm{T}}^{2}}\left(1-\left|\breve{X}_{p, i-1}\right|^{2}\right)+g_{k} \sigma^{2} .
$$

We, therefore, have

$$
p\left(\tilde{X}_{k, i} \mid X_{k}\right)=\frac{1}{2 \pi \varsigma^{2}} \exp \left(-\frac{\left|\tilde{X}_{k, i}-g_{k} X_{k}\right|^{2}}{2 \varsigma^{2}}\right) .
$$

2) IC-MRC BEME: With (16), we are ready to derive the IC-MRC BEME using $\tilde{X}_{k, i}$ as the observed signal, i.e.,

$$
\begin{aligned}
\breve{X}_{k, i, \mathrm{IC}-\mathrm{MRC}} & =E\left\{X_{k} \mid \tilde{X}_{k, i}\right\} \\
& =\sum_{S_{m} \in \Omega} S_{m} \frac{p\left(\tilde{X}_{k, i} \mid X_{k}=S_{m}\right) p\left(X_{k}=S_{m}\right)}{\sum_{S_{n} \in \Omega} p\left(\tilde{X}_{k, i} \mid X_{k}=S_{n}\right) p\left(X_{k}=S_{n}\right)} .
\end{aligned}
$$

For BPSK signals, we have

$$
\breve{X}_{k, i, \mathrm{BPSK}, \mathrm{IC}-\mathrm{MRC}}=\tanh \left(\frac{\lambda_{i}^{\mathrm{a}}(k)}{2}+\frac{g_{k} \tilde{X}_{k, i}}{\varsigma^{2}}\right) .
$$

For QPSK signals with a Gray mapping rule of $S_{k}=(1 / \sqrt{2}) \times$ $\left[\left(2 c_{k}^{1}-1\right)+j\left(2 c_{k}^{2}-1\right)\right]$, we use the $a$ priori $\mathrm{pmf}$ in $(6)$ and obtain

$$
\begin{aligned}
\breve{X}_{k, i, \mathrm{QPSK}, \mathrm{IC}-\mathrm{MRC}}= & \frac{1}{\sqrt{2}}\left[\tanh \left(\frac{\lambda_{i}^{\mathrm{a}}(k, 1)}{2}+\frac{\operatorname{Re}\left(g_{k} \tilde{X}_{k, i}\right)}{\sqrt{2} \varsigma^{2}}\right)\right. \\
& \left.+j \tanh \left(\frac{\lambda_{i}^{\mathrm{a}}(k, 2)}{2}+\frac{\operatorname{Im}\left(g_{k} \tilde{X}_{k, i}\right)}{\sqrt{2} \varsigma^{2}}\right)\right] .
\end{aligned}
$$

The BEME for other modulation schemes, e.g., eight-phase shift keying (8PSK) and $M$-ary quadrature amplitude modulation
TABLE I

BAyESIAN IC-MRC TURBo RECEIVER ALgorithm

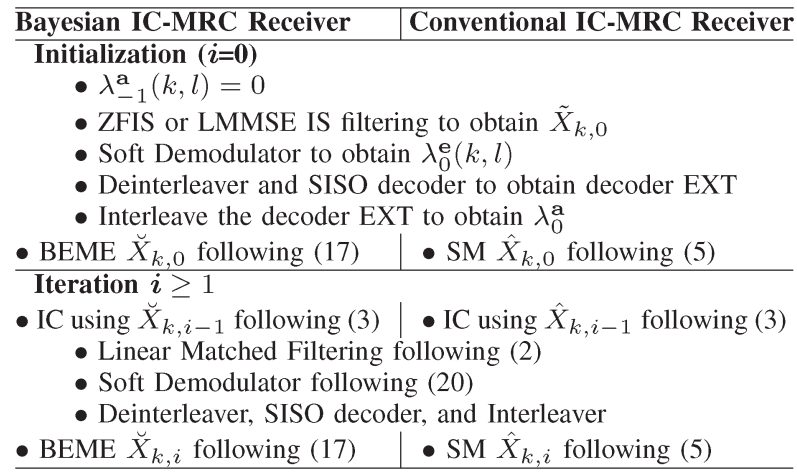

( $M$-QAM), can be derived following a similar procedure. We give the expression for 8PSK signals in Appendix B.

\section{Soft Demodulator}

The soft demodulator calculates the updated LLR of the coded bits, i.e., the extrinsic metric values, using $\tilde{X}_{k, i}$, i.e.,

$$
\begin{aligned}
\lambda_{i}^{\mathrm{e}}(k, l) & =\Lambda_{i}(k, l)-\lambda_{i-1}^{\mathrm{a}}(k, l) \\
& =\log \frac{p\left(c_{k}^{l}=1 \mid \tilde{X}_{k, i}\right)}{p\left(c_{k}^{l}=0 \mid \tilde{X}_{k, i}\right)}-\log \frac{p\left(c_{k}^{l}=1\right)}{p\left(c_{k}^{l}=0\right)} \\
& =\log \frac{\sum_{S_{k} \in \Omega^{+}} p\left(\tilde{X}_{k, i} \mid S_{k}\right) p\left(S_{k}\right)}{\sum_{S_{k} \in \Omega^{-}} p\left(\tilde{X}_{k, i} \mid S_{k}\right) p\left(S_{k}\right)}-\log \frac{p\left(c_{k}^{l}=1\right)}{p\left(c_{k}^{l}=0\right)} \\
& =\log \frac{\sum_{S_{k} \in \Omega^{+}} p\left(\tilde{X}_{k, i} \mid S_{k}\right) \prod_{\substack{j=1 \\
j \neq l}}^{\log _{2} M} p\left(c_{k}^{j}\right)}{\sum_{S_{k} \in \Omega^{-}} p\left(\tilde{X}_{k, i} \mid S_{k}\right)}
\end{aligned}
$$

where $\lambda^{E}, \Lambda$, and $\lambda^{\mathrm{a}}$, respectively, denote the extrinsic, a posteriori, and $a$ priori LLRs, and $p\left(c_{k}^{l}\right)$ is the estimated a priori pmf of $c_{k}^{l}$ given in (7). At the initialization (iteration 0 ), $p\left(c_{k}^{l}\right)=1 / 2$, and $\lambda_{-1}^{\mathrm{a}}(k, l)=0$. $\Omega^{+}=\left\{S_{k}: c_{k}^{l}=1\right\}$ denotes the subset of modulation symbols whose $l$ th bit is 1 , and $\Omega^{-}=\left\{S_{k}: c_{k}^{l}=0\right\}$ denotes the signal subset whose $l$ th bit is 0 .

\section{E. Summary of the Complete Receiver Signal Processing Steps}

We summarize the signal processing algorithms of the Bayesian IC-MRC turbo receiver in Table I. For comparison, the operation of the conventional receiver is also included in the same table. It can be seen that the major difference between the two receivers lies in the signal estimation step. The rest of the processing, such as IC, linear filtering, and soft demodulation, are the same.

\section{F. Proposed Receiver and the Sum-Product Principle}

The difference between our BEME in (17) and that in [21] and [22] is that we have improved the original EM estimate by using the estimated a priori probability from the SISO decoders rather than simply assuming an equal a priori probability. As pointed in [13, ch. 10 and 11], the use of prior information will always improve the estimation accuracy. 
On the other hand, not only the EXT from the decoder but also the decision statistics from the detector is needed to compute the BEME. Therefore, the sum-product principle has not been followed strictly. Nonetheless, performance improvement can be achieved from the BEME, as we will demonstrate in Section V. This could be due to the fact that the sum-product principle assumes Gaussian interfering signals in the IC-based linear detector. As the assumption is not true, the IC detection could be improved by not exactly following the sum-product principle.

The BEME, in general, cannot be obtained by directly replacing the EXT in the SM estimate with the a posteriori LLR. However, for BPSK and QPSK signals, only one bit is mapped to the real and imaginary parts, and the residual interference and noise are circularly symmetric complex Gaussian; we, therefore, have the following:

$$
p\left\{X_{k} \mid \tilde{X}_{k, i}\right\}=p\left\{c_{k} \mid \tilde{X}_{k, i}\right\}
$$

for BPSK, and

$$
\begin{aligned}
p\left\{X_{k} \mid \tilde{X}_{k, i}\right\}= & p\left\{c_{k}^{1}, c_{k}^{2} \mid \tilde{X}_{k, i, \operatorname{Re}}, \tilde{X}_{k, i, \operatorname{Im}}\right\} \\
= & p\left\{c_{k}^{1} \mid c_{k}^{2}, \tilde{X}_{k, i, \operatorname{Re}}, \tilde{X}_{k, i, \operatorname{Im}}\right\} \\
& \times p\left\{c_{k}^{2} \mid \tilde{X}_{k, i, \operatorname{Re}}, \tilde{X}_{k, i, \operatorname{Im}}\right\} \\
= & p\left\{c_{k}^{1} \tilde{X}_{k, i, \operatorname{Re}}\right\} p\left\{c_{k}^{2} \mid \tilde{X}_{k, i, \operatorname{Im}}\right\}
\end{aligned}
$$

for QPSK. Hence, their BEME can be obtained by directly replacing the EXT with the posterior LLR in the SM estimate, as suggested in (18) and (19).

For higher order modulation signals, e.g., 8PSK and 16-QAM, more than one bit are mapped to the real and imaginary parts. Hence

$$
p\left\{X_{k} \mid \tilde{X}_{k, i}\right\} \neq \prod_{l=1}^{\log _{2} M} p\left\{c_{k}^{l} \mid \tilde{X}_{k, i}\right\}
$$

and the BEME has to be computed following (17) instead of replacing the EXT with the posterior LLR in the SM estimation in (5), although by doing the latter, some performance gain may also be obtained, as reported in [23]. Therefore, the sum-product principle is followed strictly.

The BEME, in general, requires higher computational complexity than the SM estimation. This complexity increase, as demonstrated in Section V, leads to even better performance at the second iteration than the conventional receiver at the fifth iteration. Considering the amount of computation in each iteration, which includes IC, linear filtering, interference estimation, soft demodulation, and SISO decoding, we can easily conclude that great complexity saving is achieved by using the Bayesian receiver.

\section{BAYESIAN LMMSE-IC TURBO RECEIVER}

Similar to the conventional LMMSE-IC turbo receivers [6], [24], LMMSE filtering can be applied in (2) to further suppress the residual interference in a Bayesian turbo receiver. As proven in [15], the LMMSE filter can be modeled by an equivalent AWGN channel. We can, therefore, obtain the BEME of the signals for the LMMSE-IC turbo receiver in a very straightforward manner. The LMMSE filter $\mathbf{F}_{k, i}$ minimizes the mean square error between the transmitted signal $X_{k}$ and the filter output $\tilde{X}_{k, i}=\mathbf{F}_{k, i}^{H} \tilde{\mathbf{R}}_{k, i}$ and is obtained from the Wiener-Hopf equation as follows:

$$
\begin{aligned}
\mathbf{F}_{k, i} & =E\left\{\tilde{\mathbf{R}}_{k, i} \tilde{\mathbf{R}}_{k, i}^{H}\right\}^{-1} E\left\{X_{k}^{*} \tilde{\mathbf{R}}_{k, i}\right\} \\
& =\left(\mathbf{H} \boldsymbol{\Gamma}_{k, i} \mathbf{H}^{H}+2 \sigma^{2} \mathbf{I}\right)^{-1} \mathbf{H}_{k}
\end{aligned}
$$

where $\boldsymbol{\Gamma}_{k, i}$ is a diagonal matrix with elements

$$
\gamma_{p, i}=\left\{\begin{array}{cc}
1, & p=k \\
\sum_{X_{p} \in \Omega} p\left\{X_{p}\right\}\left|X_{p}\right|^{2}+\left|\breve{X}_{p, i-1}\right|^{2}, & p \neq k \\
-\breve{X}_{p, i-1}^{*} \hat{X}_{p, i}-\breve{X}_{p, i-1} \hat{X}_{p, i}^{*} &
\end{array}\right.
$$

with $\hat{X}_{p, i}$ and $\breve{X}_{p, i}$ being the SM and the BEME of $X_{p}$, respectively. Note that due to the use of the Bayesian estimate in the IC process, the computation of $\gamma_{p \neq k, i}$ here is different from that in the conventional LMMSE-IC receivers.

The LMMSE-IC decision statistic is given by

$$
\tilde{X}_{k, i}=\mathbf{F}_{k, i}^{H} \tilde{\mathbf{R}}_{k, i}=\mu_{k, i} X_{k}+\eta_{k, i}
$$

which is an equivalent AWGN model with [15]

$$
\begin{aligned}
& \mu_{k, i}=E\left\{\tilde{X}_{k, i} X_{k}^{*}\right\}=\left\{\mathbf{H}^{H}\left[\mathbf{H} \boldsymbol{\Gamma}_{k, i} \mathbf{H}^{H}+2 \sigma^{2} \mathbf{I}\right]^{-1} \mathbf{H}\right\}_{k k} \\
& \nu_{k, i}^{2}=\operatorname{Var}\left\{\tilde{\mathrm{X}}_{\mathrm{k}, \mathrm{i}}\right\}=\mu_{\mathrm{k}, \mathrm{i}}-\mu_{\mathrm{k}, \mathrm{i}}^{2}
\end{aligned}
$$

and $\{\cdot\}_{i j}$ denotes the element at the $i$ th row and the $j$ th column.

We, therefore, have

$$
p\left(\tilde{X}_{k, i} \mid X_{k}\right)=\frac{1}{2 \pi \nu_{k, i}^{2}} \exp \left(-\frac{\left|\tilde{X}_{k, i}-\mu_{k, i} X_{k}\right|^{2}}{2 \nu_{k, i}^{2}}\right) .
$$

With (26), we can derive the LMMSE-IC BEME using $\tilde{X}_{k, i}$ as the observed data, i.e.,

$$
\begin{aligned}
\breve{X}_{k, i, \mathrm{LMMSE}-\mathrm{IC}} & =E\left\{X_{k} \mid \tilde{X}_{k, i}\right\} \\
& =\sum_{S_{m} \in \Omega} S_{m} \frac{p\left(\tilde{X}_{k, i} \mid X_{k}=S_{m}\right) p\left(X_{k}=S_{m}\right)}{\sum_{S_{n} \in \Omega} p\left(\tilde{X}_{k, i} \mid X_{k}=S_{n}\right) p\left(X_{k}=S_{n}\right)} .
\end{aligned}
$$

Specifically, we have

$$
\breve{X}_{k, i, \text { BPSK }, \text { LMMSE-IC }}=\tanh \left(\frac{\lambda_{i}^{\mathrm{a}}(k)}{2}+\frac{\mu_{k, i} \tilde{X}_{k, i}}{\nu_{k, i}^{2}}\right)
$$

for BPSK signals and

$$
\begin{aligned}
\breve{X}_{k, i, \mathrm{QPSK}, \mathrm{LMMSE}-\mathrm{IC}} & =\frac{1}{\sqrt{2}}\left[\tanh \left(\frac{\lambda_{i}^{\mathrm{a}}(k, 1)}{2}+\frac{\operatorname{Re}\left(\mu_{\mathrm{k}, \mathrm{i}} \tilde{\mathrm{X}}_{\mathrm{k}, \mathrm{i}}\right)}{\sqrt{2} \nu_{\mathrm{k}, \mathrm{i}}^{2}}\right)\right. \\
+ & \left.j \tanh \left(\frac{\lambda_{i}^{\mathrm{a}}(k, 2)}{2}+\frac{\operatorname{Im}\left(\mu_{\mathrm{k}, \mathrm{i}} \tilde{\mathrm{X}}_{\mathrm{k}, \mathrm{i}}\right)}{\sqrt{2} \nu_{\mathrm{k}, \mathrm{i}}^{2}}\right)\right]
\end{aligned}
$$

for QPSK signals.

The BEME for other modulation schemes can be derived in a similar manner. We give the BEME of 8PSK signals in Appendix B.

The Bayesian LMMSE-IC turbo receiver operation is summarized in Table II. For comparison, the conventional LMMSE-IC receiver algorithms are also included in Table II. The major differences between the proposed and conventional LMMSE-IC turbo receivers lie in the signal estimation and LMMSE filter coefficient calculation; however, the remaining steps are the same. 
TABLE II

BAyesian LMMSE-IC TURbo ReCEIVER ALgorithm
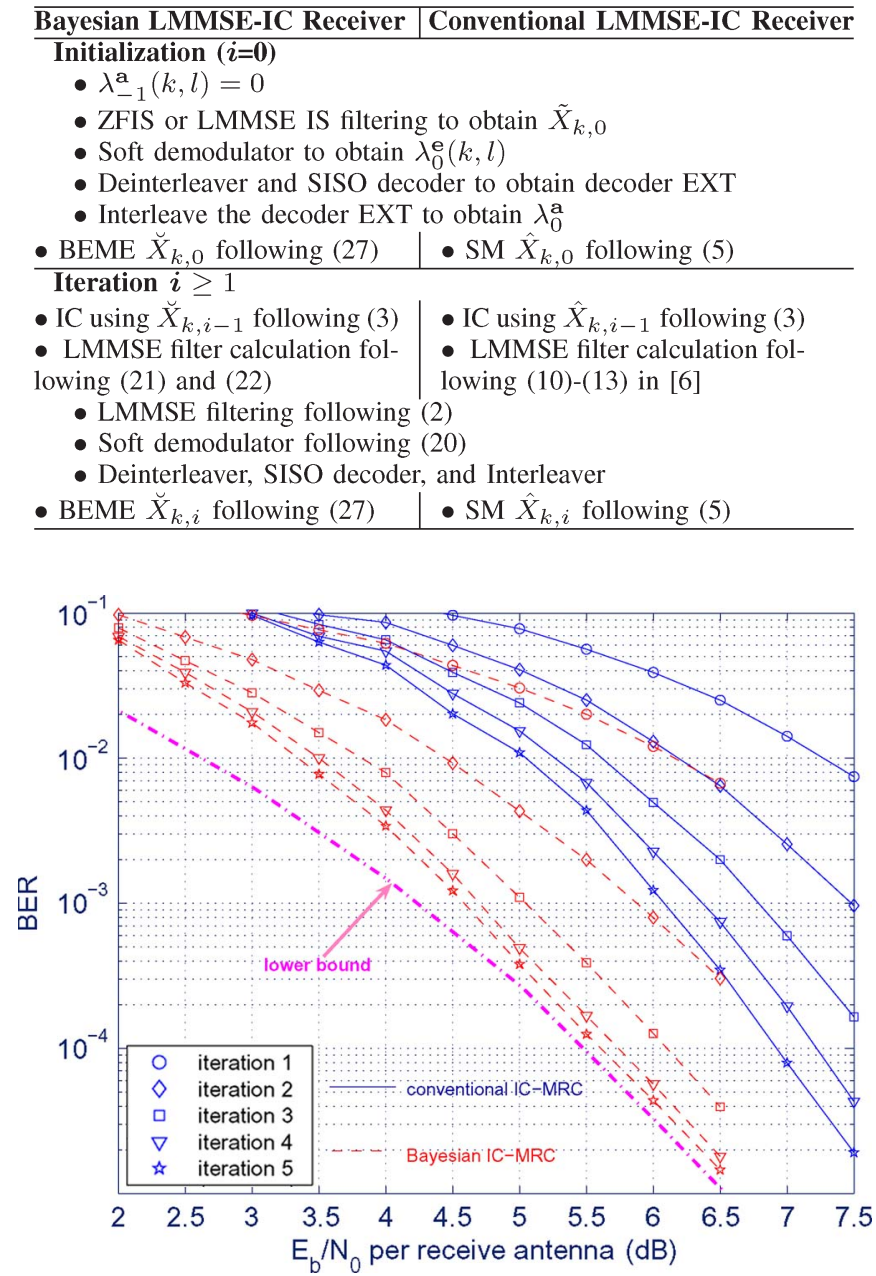

Fig. 3. BER performance of the Bayesian IC-MRC receiver, $8 \times 4$ GSTBC, QPSK, $R_{c}=1 / 2$, and $K=3$.

\section{NUMERICAL RESULTS}

In this section, we present the numerical results and demonstrate the superior performance of the proposed Bayesian turbo receivers by using the EXIT chart and the BER.

In all the simulations, the rate $R_{c}=1 / 2$, constraint length $K=3$ convolutional code (CC) with generation function $g=(5,7)_{\text {octal }}$ is used as the mother code. The puncturing pattern in [25] and [26] is followed to generate the higher rate code. A log-MAP decoding algorithm is used in the SISO decoders. The HCA is adopted for the BER simulations, and the VCA is used for the EXIT chart analysis. For OFDM, we set the FFT size to 64 and assign 48 subcarriers for data transmission following IEEE 802.11a [27]. The channel is assumed quasi-static within each packet but randomly and independently varies between the packets. Uniform power delay profiles with 16 independent identically distributed (i.i.d.) complex Gaussian taps are used for the uncorrelated spatial channels corresponding to each transmit-receive antenna pair.

\section{A. BER Performance}

We first present in Fig. 3 the BER performance of the IC-MRC Bayesian MMSE receiver at iterations $i=1, \ldots, 5$ for an $8 \times 4$ GSTBC system with $R_{c}=1 / 2$ and QPSK modulation. Zero-forcing

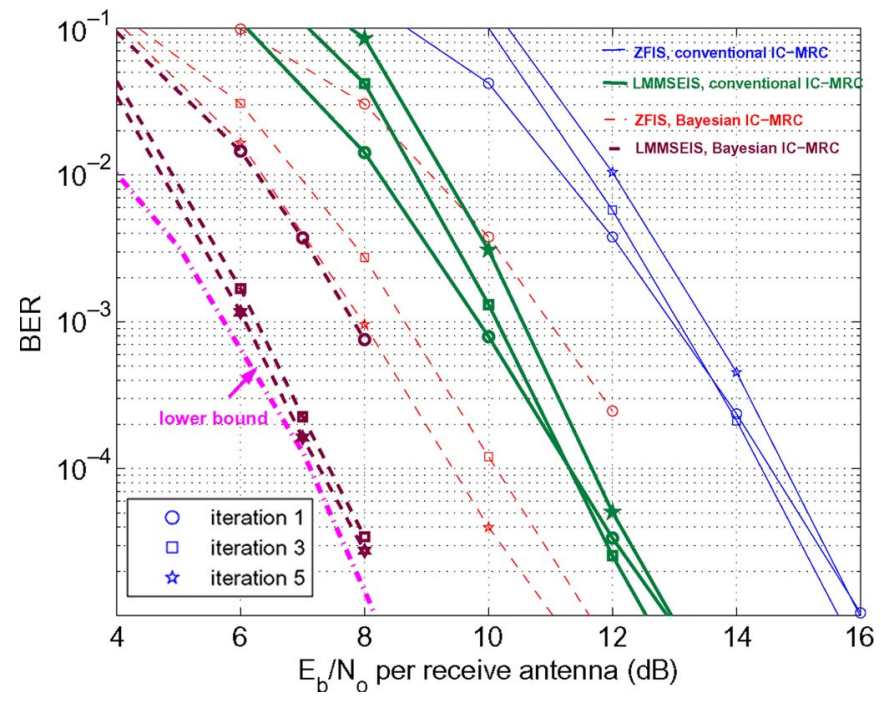

Fig. 4. BER performance comparison of the Bayesian IC-MRC and conventional IC-MRC receivers, ZFIS, LMMSE IS, $8 \times 4$ GSTBC, QPSK, $R_{c}=3 / 4$, and $K=3$.

interference suppression (ZFIS) is used for the initialization. For comparison, the conventional IC-MRC turbo receiver performance and the lower bound of the perfect IC are also depicted. Several observations can be made from Fig. 3. First, the Bayesian receiver has superior performance over the conventional counterpart. Its second iteration has better performance than the conventional receiver's fifth iteration at low-to-medium SNR values and the same performance at a high SNR. This is because of the improved accuracy in the BMMSE interference estimation, as shown in Section III. With the same number of iterations, an SNR gain of $1.2 \mathrm{~dB}$ can be achieved from the Bayesian receiver at iteration five, and $\mathrm{BER}=10^{-5}$. The convergence of the Bayesian receiver to the lower bound appears at $\mathrm{SNR}=4.5 \mathrm{~dB}$, corresponding to $\mathrm{BER}=6 \times 10^{-4}$, whereas the conventional receiver cannot converge to the lower bound in the SNR range in our simulation setups.

The performance advantage of the Bayesian receiver is more obvious when a punctured code is used, as exhibited in the BER simulation results in Fig. 4 for an $R_{c}=3 / 4$ QPSK $8 \times 4$ GSTBC system. BER versus $E_{b} / N_{0}$ curves at iterations 1,3 , and 5 are presented for four receivers, namely, conventional IC-MRC receivers with both ZFIS and LMMSE IS initialization and Bayesian IC-MRC receivers with ZFIS and LMMSE IS initialization. From Fig. 4, it is clear that the BER of the conventional receivers gets worse with an increasing number of iterations. We believe that this is due to puncturing in the $\mathrm{CC}$ that severely degrades the accuracy in the EXT. The Bayesian receivers, with the compensation of the detector's decision statistic, have a decreasing BER with an increasing number of iterations. A 5-dB gain can be achieved over the conventional receivers with both ZFIS and LMMSE IS initialization, and more importantly, the Bayesian receiver with LMMSE IS initialization reaches the lower bound at $\mathrm{BER}=10^{-3}$ and $\mathrm{SNR}=6 \mathrm{~dB}$.

The BER performance of the Bayesian LMMSE-IC receiver is depicted in Fig. 5 for an $8 \times 8$ VBLAST system with $R_{c}=3 / 4$ 8PSK. Similar to the IC-MRC Bayesian receivers, its second-iteration performance is better than that of the fifth iteration in the conventional receiver at low-to-medium SNR values due to the improved accuracy in the interference estimation, and at a high SNR, the performance approaches that of the conventional receiver due to the dominance of the a priori information in the Bayesian estimate. For all five iterations, the Bayesian receiver achieves at least a $1-\mathrm{dB}$ gain over 


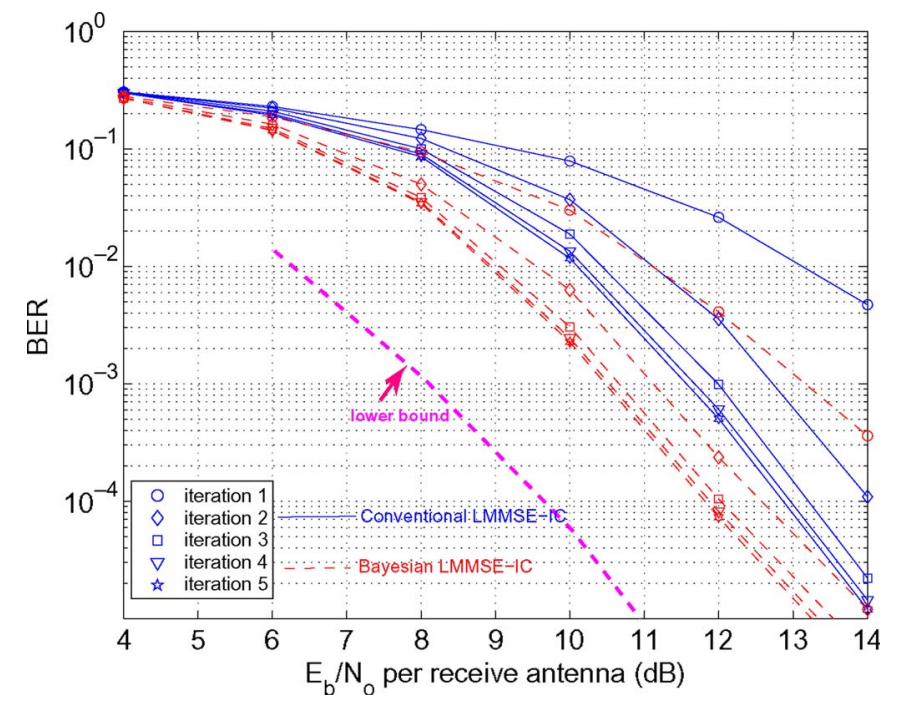

Fig. 5. BER performance comparison of the Bayesian LMMSE-IC and conventional LMMSE-IC receivers, $8 \times 8$ VBLAST, 8 PSK, $R_{c}=3 / 4$, and $K=3$.

the conventional one. When compared with the lower bound, however, there is still an approximately $2-\mathrm{dB}$ gap to bridge.

\section{B. EXIT Chart Analysis}

We now demonstrate the superior performance of the Bayesian receivers through the EXIT chart analysis. The $4 \times 4$ fixed channel matrix that is given in [28] is used. The a priori information input to the detector is assumed to be Gaussian distributed, with $\lambda_{i}^{\mathrm{a}} \mid C_{i} \sim$ $\mathcal{N}\left(C_{i} \sigma_{\mathrm{a}}^{2} / 2, \sigma_{\mathrm{a}}^{2}\right)$, as given in [29]. The corresponding input mutual information $I^{\mathrm{a}}$ is calculated from $\sigma_{\mathrm{a}}^{2}$ using the following mapping function [29]:

$I^{\mathrm{a}}=J\left(\sigma_{\mathrm{a}}^{2}\right)=1-\int_{-\infty}^{\infty} \frac{1}{\sqrt{2 \pi \sigma_{\mathrm{a}}^{2}}} e^{-\frac{\left(z-\frac{\sigma_{\mathrm{a}}^{2}}{2}\right)^{2}}{2 \sigma_{\mathrm{a}}^{2}}}$

$$
\times \log _{2}[1+\exp (-z)] d z .
$$

For the conventional receivers, we use the a priori information, i.e., the EXT, for the interference estimation. For the proposed Bayesian receivers, we use the EXT and the detector decision statistic for the interference estimation. The Gaussian-distributed EXT is generated the same way as in the conventional detectors. The decision statistic is generated from the EXT following the steps outlined as follows.

1) Obtain the decoder output mutual information from the EXT using $I_{\mathrm{dec}}^{\mathrm{e}}=J\left(\sigma_{\mathrm{e}}^{2}\right)$.

2) Obtain the decoder's input mutual information from the inverse of the decoder EXIT function as $I_{\mathrm{dec}}^{\mathrm{a}}=T_{\mathrm{dec}}^{-1}\left(I_{\mathrm{dec}}^{\mathrm{e}}\right)$, where $T_{\mathrm{dec}}$ denotes the EXIT function of the decoder.

3) Obtain the decoder's input a priori information as $\sigma_{\mathrm{a}}^{2}=$ $J^{-1}\left(I_{\mathrm{dec}}^{\mathrm{a}}\right)$.

4) Obtain the decision statistic from the decoder's input a priori information.

IC is then performed, and the detector output mutual information is calculated from the output extrinsic LLR as [30]

$$
I^{\mathrm{e}}\left(L_{\mathrm{e}} ; C\right)=1-\frac{1}{N} \sum_{n=1}^{N} \log _{2}\left[1+\exp \left(-C_{n} \lambda_{n}^{\mathrm{e}}\right)\right] .
$$

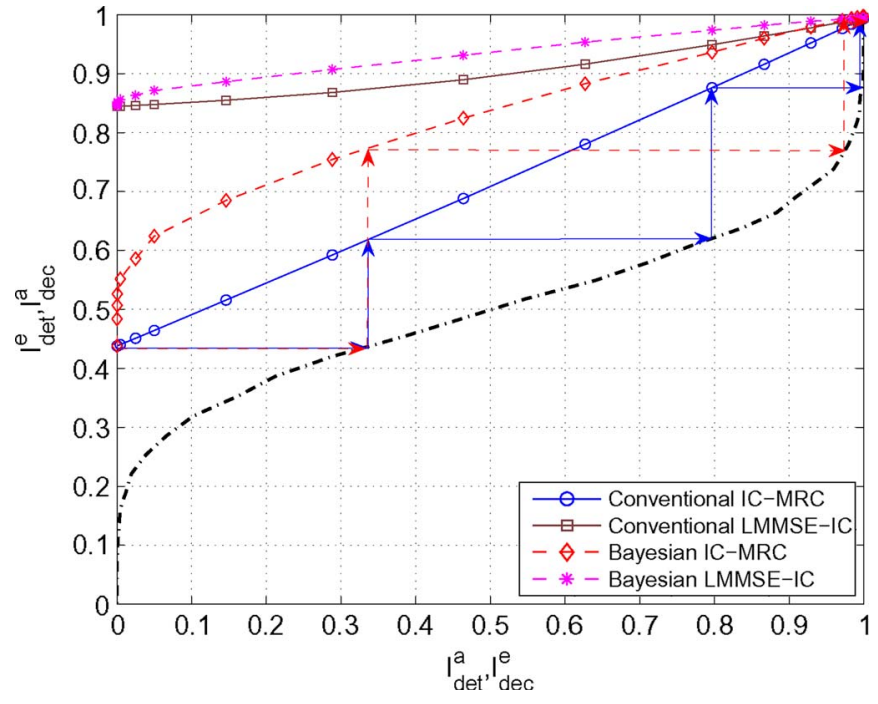

Fig. 6. Mutual information transfer function comparison of the conventional and Bayesian receivers and the decoding path for the turbo receivers with $R_{c}=1 / 2$ generation function $g=(5,7)_{\text {octal }}$ CC. Static channel, QPSK modulation, and $\sigma^{2}=0.1256$.

Fig. 6 depicts the EXIT chart of the proposed Bayesian and conventional turbo receivers with QPSK modulated signals and the AWGN power of $\sigma^{2}=0.1256$. As expected, the Bayesian receiver outperforms its conventional counterpart, and the Bayesian LMMSE-IC receiver has the best performance.

From Fig. 6, we also observe that the EXIT functions of the conventional and Bayesian receivers merge at the starting point of $I_{\mathrm{det}}^{\mathrm{a}}=0$ and the ending point of $I_{\mathrm{det}}^{\mathrm{a}}=1$. For $0<I_{\mathrm{det}}^{\mathrm{a}}<1$, the smaller the input $I_{\text {det }}^{\mathrm{a}}$, the larger the difference between the Bayesian and the conventional detectors' output $I_{\mathrm{det}}^{\mathrm{e}}$ values. This is expected, as when $I_{\mathrm{det}}^{\mathrm{a}}=0$, there is no a priori information available, and the two receivers have the same performance; at small input $I_{\mathrm{det}}^{\mathrm{a}}$ 's, the BMMSE interference estimation accuracy is greatly improved by using the additional detector decision statistic, and when $I_{\text {det }}^{\mathrm{a}}$ increases, the $a$ priori probability becomes increasingly dominant in the BMMSE estimate, making it increasingly close to the a priori estimate.

We also illustrate the convergence behavior of the proposed and conventional IC-MRC turbo receivers in Fig. 6. From the trajectories of the conventional and Bayesian receivers, we can see that fewer iterations are needed by the Bayesian receiver to achieve the same performance. Furthermore, performance improvement from the initial iterations in the Bayesian receiver is substantial, which is consistent with the BER results in Section V-A.

\section{CONCLUSION}

A class of Bayesian MMSE turbo receivers has been presented for coded MIMO systems. Using the EM algorithm, we have proposed a novel application of the turbo principle by making use of the EXT in the posterior Bayesian MMSE signal estimation. We have shown that the Bayesian MMSE estimate of the transmitted signals is a function of the linear detector decision statistic and the EXT from the SISO decoder. The Bayesian MMSE estimate effectively compensates for the inaccuracy that is experienced by the prior SM estimation using only the EXT in conventional turbo receivers. This contributes to the SNR gains at the same BER performance and the fewer number of iterations that are needed to achieve convergence. The EXIT chart analysis has further proved that a significant improvement can be obtained in the detector output mutual information over the conventional receiver. 


\section{APPENDIX A}

MEAN AND VARIANCE OF THE INTERFERENCE AT THE IC-MRC OUTPUT

Defining $\rho_{k p}=\mathbf{H}_{k}^{H} \mathbf{H}_{p}$, we have

$$
\begin{aligned}
E\left\{\tilde{v}_{k}\right\} & =E\left\{\sum_{\substack{p=1 \\
p \neq k}}^{n_{\mathrm{T}}} \rho_{k p}\left(X_{p}-\breve{X}_{p, i-1}\right)+\mathbf{H}_{k}^{H} \mathbf{V}\right\} \\
& =\sum_{\substack{p=1 \\
p \neq k}}^{n_{\mathrm{T}}} \rho_{k p} E\left\{X_{p}-\breve{X}_{p, i-1}\right\}+\mathbf{H}_{k}^{H} E\{\mathbf{V}\}=0
\end{aligned}
$$

and

$$
\begin{aligned}
E\left\{\left|\tilde{v}_{k}\right|^{2}\right\} & =E\left\{\left|\sum_{\substack{p=1 \\
p \neq k}}^{n_{\mathrm{T}}} \rho_{k p}\left(X_{p}-\breve{X}_{p, i-1}\right)+\mathbf{H}_{k}^{H} \mathbf{V}\right|^{2}\right\} \\
& =E\left\{\left|\sum_{\substack{p=1 \\
p \neq k}}^{n_{\mathrm{T}}} \rho_{k p}\left(X_{p}-\breve{X}_{p, i-1}\right)\right|^{2}\right\}+E\left\{\left|\mathbf{H}_{k}^{H} \mathbf{V}\right|^{2}\right\}
\end{aligned}
$$

with

$$
\begin{aligned}
E\{\mid & \left.\left|\sum_{\substack{p=1 \\
p \neq k}}^{n_{\mathrm{T}}} \rho_{k p}\left(X_{p}-\breve{X}_{p, i-1}\right)\right|^{2}\right\} \\
& =E\left\{\sum_{\substack{p=1 \\
p \neq k}}^{n_{\mathrm{T}}} \sum_{\substack{q=1, q \neq k}}^{n_{\mathrm{T}}} \rho_{k p} \rho_{k q}^{*}\left(X_{p}-\breve{X}_{p, i-1}\right)\left(X_{q}^{*}-\breve{X}_{q, i-1}^{*}\right)\right\} \\
& =\sum_{\substack{p=1 \\
n_{\mathrm{T}}}}^{n_{\mathrm{T}}} \sum_{\substack{q=1, q \neq k}} E\left\{\rho_{k p} \rho_{k q}^{*}\right\} E\left[\left(X_{p}-\breve{X}_{p, i-1}\right)\left(X_{q}^{*}-\breve{X}_{q, i-1}^{*}\right)\right]
\end{aligned}
$$

under the assumption that $\rho_{k p}$ is independent of $X_{p}$ and $\breve{X}_{p, i-1}$, and

$$
E\left\{\left|\mathbf{H}_{k}^{H} \mathbf{V}\right|^{2}\right\}=2 \sigma^{2} g_{k} .
$$

For single-carrier systems in flat-fading channels, assuming that the spatial domain channels are perfectly uncorrelated, we have

$$
\begin{aligned}
E\left\{\rho_{k p} \rho_{k q}^{*}\right\}= & E\left\{\sum_{g=1}^{n_{\mathrm{R}}} H_{g, k}^{*} H_{g, p} \sum_{n=1}^{n_{\mathrm{R}}} H_{n, k} H_{n, q}^{*}\right\} \\
= & \sum_{g=1}^{n_{\mathrm{R}}} \sum_{n=1}^{n_{\mathrm{R}}} E\left\{H_{g, k}^{*} H_{g, p} H_{n, k} H_{n, q}^{*}\right\} \\
= & \sum_{g=1}^{n_{\mathrm{R}}} \sum_{n=1}^{n_{\mathrm{R}}} E\left\{H_{g, k}^{*} H_{g, p}\right\} E\left\{H_{n, k} H_{n, q}^{*}\right\} \\
& +E\left\{H_{g, k}^{*} H_{n, k}\right\} E\left\{H_{n, q}^{*} H_{g, p}\right\} \\
= & \frac{1}{n_{\mathrm{T}}^{2}} \sum_{g=1}^{n_{\mathrm{R}}} \sum_{n=1}^{n_{\mathrm{R}}} \delta(k-p) \delta(k-q)+\delta(g-n) \delta(p-q)
\end{aligned}
$$

by using the result from [31] that

$$
\mathcal{E}\left\{Z_{1}^{*} Z_{2}^{*} Z_{3} Z_{4}\right\}=\mathcal{E}\left\{Z_{1}^{*} Z_{3}\right\} \mathcal{E}\left\{Z_{2}^{*} Z_{4}\right\}+\mathcal{E}\left\{Z_{1}^{*} Z_{4}\right\} \mathcal{E}\left\{Z_{2}^{*} Z_{3}\right\}
$$

if $Z_{1}, Z_{2}, Z_{3}$, and $Z_{4}$ are zero-mean stationary complex Gaussian. Here, $\delta(\cdot)$ is the Dirac delta function.
When $k \neq p$, we have

$$
E\left\{\rho_{k p} \rho_{k q}^{*}\right\}=\frac{1}{n_{\mathrm{T}}^{2}} \sum_{g=1}^{n_{\mathrm{R}}} \sum_{n=1}^{n_{\mathrm{R}}} \delta(g-n) \delta(p-q)=n_{\mathrm{R}} n_{\mathrm{T}}^{2} \delta(p-q) .
$$

For OFDM systems, we have the following FD channel coefficients:

$$
H_{m, n, k}=\sum_{l=0}^{L-1} h_{l, n, k} \exp \left(-\frac{2 \pi}{N} m l\right)
$$

where $m=0,1,2, \ldots, N-1$ is the subcarrier index, $N$ is the FFT size, $n=1,2, \ldots, n_{\mathrm{R}}$ and $k=1,2, \ldots, n_{\mathrm{T}}$ are the receive and transmit antenna indexes, respectively, $L$ is the number of multipaths in the channel corresponding to the transmit-receive antenna pair $(k, n)$, and $h_{l, n, k}$ is the time-domain multipath coefficient. Here, we consider the uniform power delay profile in which each multipath is i.i.d. complex Gaussian with zero mean and variance $1 / L n_{\mathrm{T}}$ so that the average signal power at each receive antenna remains the same as the mapper output.

We, hence, have, for $k \neq p$

$$
\begin{aligned}
& E\left\{\rho_{k p} \rho_{k q}^{*}\right\}=E\left\{\sum_{g=1}^{n_{\mathrm{R}}} H_{m, g, k}^{*} H_{m, g, p} \sum_{n=1}^{n_{\mathrm{R}}} H_{m, n, k} H_{m, n, q}^{*}\right\} \\
& =\sum_{g=1}^{n_{\mathrm{R}}} \sum_{n=1}^{n_{\mathrm{R}}} E\left\{H_{m, g, k}^{*} H_{m, g, p} H_{m, n, k} H_{m, n, q}^{*}\right\} \\
& =\sum_{g=1}^{n_{\mathrm{R}}} \sum_{n=1}^{n_{\mathrm{R}}} E\left\{\sum_{l=0}^{L-1} h_{l, g, k}^{*} \exp \left(j \frac{2 \pi}{N} m l\right)\right. \\
& \times \sum_{t=0}^{L-1} h_{t, g, p} \exp \left(-j \frac{2 \pi}{N} m t\right) \\
& \times \sum_{i=0}^{L-1} h_{i, n, k} \exp \left(-j \frac{2 \pi}{N} m i\right) \\
& \left.\times \sum_{s=0}^{L-1} h_{s, n, q}^{*} \exp \left(j \frac{2 \pi}{N} m s\right)\right\} \\
& =\sum_{g=1}^{n_{\mathrm{R}}} \sum_{n=1}^{n_{\mathrm{R}}} \sum_{l=0}^{L-1} \sum_{i=0}^{L-1} \sum_{t=0}^{L-1} \sum_{s=0}^{L-1} E\left\{h_{l, g, k}^{*} h_{i, n, k} h_{t, g, p} h_{s, n, q}^{*}\right\} \\
& \times \exp \left(j \frac{2 \pi}{N} m(l-i-t+s)\right) \\
& =\sum_{g=1}^{n_{\mathrm{R}}} \sum_{n=1}^{n_{\mathrm{R}}} \sum_{l=0}^{L-1} \sum_{i=0}^{L-1} \sum_{t=0}^{L-1 L-1} \sum_{s=0}^{L}\left[E\left\{h_{l, g, k}^{*} h_{i, n, k}\right\}\right. \\
& \times E\left\{h_{t, g, p} h_{s, n, q}^{*}\right\} \\
& +E\left\{h_{s, n, q}^{*} h_{i, n, k}\right\} \\
& \left.\times E\left\{h_{l, g, k}^{*} h_{t, g, p}\right\}\right] \\
& \times \exp \left(j \frac{2 \pi}{N} m(l-i-t+s)\right) \\
& =\frac{1}{n_{\mathrm{T}}^{2} L^{2}} \sum_{g=1}^{n_{\mathrm{R}}} \sum_{n=1}^{n_{\mathrm{R}}} \sum_{l=0}^{L-1} \sum_{i=0}^{L-1} \sum_{t=0}^{L-1} \sum_{s=0}^{L-1}[\delta(l-i) \delta(g-n) \\
& \times \delta(t-s) \delta(p-q) \\
& \times+\delta(s-i) \delta(k-q) \\
& \times \delta(l-t) \delta(k-p)] \\
& \times \exp \left(j \frac{2 \pi}{N} m(l-i-t+s)\right) \\
& =\frac{n_{\mathrm{R}}}{n_{\mathrm{T}}^{2}} \delta(p-q) \text {. }
\end{aligned}
$$


TABLE III

8PSK GRAY MAPPING TABLE

\begin{tabular}{|c|c|c|}
\hline INPUT BITS $\left(b_{1} b_{2}\right)$ & I-OUT & Q-OUT \\
\hline 000 & a & b \\
\hline 001 & b & a \\
\hline 011 & $-b$ & a \\
\hline 010 & -a & b \\
\hline 110 & $-a$ & $-b$ \\
\hline 111 & - b & - a \\
\hline 101 & b & -a \\
\hline 100 & a & -b \\
\hline \\
$a=\frac{\sqrt{2+\sqrt{2}}}{2}, b=\frac{\sqrt{2-\sqrt{2}}}{2}$
\end{tabular}

We then obtain the composite interference and the AWGN noise power for the narrow-band single-carrier systems and the wideband OFDM systems as follows:

$$
\varsigma^{2}=\frac{1}{2} E\left\{\left|\tilde{\mu}_{k, i}\right|^{2}\right\}=\frac{1}{2} \sum_{\substack{p=1 \\ p \neq k}}^{n_{\mathrm{T}}} \frac{n_{\mathrm{R}}}{n_{\mathrm{T}}^{2}}\left(1-\left|\breve{X}_{p, i-1}\right|^{2}\right)+g_{k} \sigma^{2} .
$$

\section{APPENDIX B \\ BEME FOR 8PSK SIGNALS}

For 8PSK signals following the Gray mapping rule in Table III, we obtain the BEME in the IC-MRC receiver as follows:

$$
\begin{aligned}
& \breve{X}_{k, i, 8 \text { PSK }, \text { IC-MRC }} \\
& =\frac{a \cosh \left(\dot{x}_{1}\right) \sinh \left(\dot{x}_{3}\right)+b \cosh \left(\dot{x}_{2}\right) \sinh \left(\dot{x}_{4}\right) e^{\lambda_{i}^{\mathrm{a}}(k, 3)}}{\cosh \left(\dot{x}_{1}\right) \cosh \left(\dot{x}_{3}\right)+\cosh \left(\dot{x}_{2}\right) \cosh \left(\dot{x}_{4}\right) e^{\lambda_{i}^{\mathrm{a}}(k, 3)}} \\
& \quad+j \frac{a \cosh \left(\dot{x}_{4}\right) \sinh \left(\dot{x}_{2}\right) e^{\lambda_{i}^{\mathrm{a}}(k, 3)}+b \cosh \left(\dot{x}_{3}\right) \sinh \left(\dot{x}_{1}\right)}{\cosh \left(\dot{x}_{1}\right) \cosh \left(\dot{x}_{3}\right)+\cosh \left(\dot{x}_{2}\right) \cosh \left(\dot{x}_{4}\right) e^{\lambda_{\mathrm{a}}^{i-1}(k, 3)}}
\end{aligned}
$$

where

$$
\begin{aligned}
& \dot{x}_{1}=\frac{b g_{k} \tilde{X}_{k, i, \operatorname{Im}}}{\varsigma^{2}}-\frac{\lambda_{i}^{\mathrm{a}}(k, 1)}{2} \\
& \dot{x}_{2}=\frac{a g_{k} \tilde{X}_{k, i, \operatorname{Im}}}{\varsigma^{2}}-\frac{\lambda_{i}^{\mathrm{a}}(k, 1)}{2} \\
& \dot{x}_{3}=\frac{a g_{k} \tilde{X}_{k, i, \mathrm{Re}}}{\varsigma^{2}}-\frac{\lambda_{i}^{\mathrm{a}}(k, 2)}{2} \\
& \dot{x}_{4}=\frac{b g_{k} \tilde{X}_{k, i, \mathrm{Re}}}{\varsigma^{2}}-\frac{\lambda_{i}^{\mathrm{a}}(k, 2)}{2}
\end{aligned}
$$

with $a=\sqrt{2+\sqrt{2}} / 2$ and $b=\sqrt{2-\sqrt{2}} / 2$, and $\varsigma^{2}$ is defined in (30).

For the LMMSE-IC receiver, the BEME of the 8PSK signal is calculated following (31) as well, but with $\dot{x}_{i}, i=1, \ldots, 4$, which is defined as follows:

$$
\begin{aligned}
& \dot{x}_{1}=\frac{b \mu_{k, i} \tilde{X}_{k, i, \operatorname{Im}}}{\nu_{k, i}^{2}}-\frac{\lambda_{i}^{\mathrm{a}}(k, 1)}{2} \\
& \dot{x}_{2}=\frac{a \mu_{k, i} \tilde{X}_{k, i, \operatorname{Im}}}{\nu_{k, i}^{2}}-\frac{\lambda_{i}^{\mathrm{a}}(k, 1)}{2} \\
& \dot{x}_{3}=\frac{a \mu_{k, i} \tilde{X}_{k, i, \operatorname{Re}}}{\nu_{k, i}^{2}}-\frac{\lambda_{i}^{\mathrm{a}}(k, 2)}{2} \\
& \dot{x}_{4}=\frac{b \mu_{k, i} \tilde{X}_{k, i, \operatorname{Re}}}{\nu_{k, i}^{2}}-\frac{\lambda_{i}^{\mathrm{a}}(k, 2)}{2}
\end{aligned}
$$

and $\mu_{k, i}$ and $\nu_{k, i}$ are defined in (24) and (25), respectively.

\section{ACKNOWLEDGMENT}

The authors would like to thank the reviewers for their valuable comments and Prof. L. K. Rasmussen for discussions.

\section{REFERENCES}

[1] G. J. Foschini, "Layered space-time architecture for wireless communications in a fading environment when using multi-element antennas," Bell Labs Tech. J., vol. 1, no. 2, pp. 41-59, 1996.

[2] G. J. Foschini, G. D. Golden, R. A. Valenzuela, and P. W. Wolnianski, "Simplified processing for high spectral efficiency wireless communication employing multi-element arrays," IEEE J. Sel. Areas Commun., vol. 17, no. 11, pp. 1841-1852, Nov. 1999.

[3] S. M. Alamouti, "A simple transmit diversity technique for wireless communications," IEEE J. Sel. Areas Commun., vol. 16, no. 8, pp. 1451-1458, Oct. 1998.

[4] V. Tarokh, H. Jafarkhani, and A. R. Calderbank, "Space-time block codes from orthogonal designs," IEEE Trans. Inf. Theory, vol. 45, no. 5, pp. 1456-1467, Jul. 1999.

[5] S. Sun, Y. Wu, Y. Li, and T. T. Tjhung, "A novel iterative receiver for coded MIMO OFDM systems," in Proc. IEEE Int. Conf. Commun., Jun. 2004, vol. 4, pp. 2473-2477.

[6] B. Lu and X. Wang, "Iterative receivers for multiuser space-time coding systems," IEEE J. Sel. Areas Commun., vol. 18, no. 11, pp. 2322-2335, Nov. 2000.

[7] M. Sellathurai and S. Haykin, "Turbo-BLAST for wireless communications: Theory and experiments," IEEE Trans. Signal Process., vol. 50, no. 10 , pp. 2538-2546, Oct. 2002.

[8] M. Sellathurai and S. Haykin, "Turbo-BLAST: Performance evaluation in correlated Rayleigh-fading environment," IEEE J. Sel. Areas Commun., vol. 21, no. 3, pp. 340-349, Apr. 2003.

[9] M. Sellathurai and S. Haykin, "T-BLAST for wireless communications: First experimental results," IEEE Trans. Veh. Technol., vol. 52, no. 3, pp. 530-535, May 2003.

[10] E. Biglieri, A. Nordio, and G. Taricco, "Iterative receiver interfaces for coded multiple-antenna signaling," in Proc. 2nd Int. Workshop Signal Process. Wireless Commun., Jun. 2004, pp. 1-12.

[11] J. Boutros and G. Caire, "Iterative multiuser joint decoding: Unified framework and asymptotic analysis," IEEE Trans. Inf. Theory, vol. 48, no. 7, pp. 1772-1793, Jul. 2002.

[12] F. Kschischang, B. Frey, and H.-A. Loeliger, "Factor graphs and the sumproduct algorithm," IEEE Trans. Inf. Theory, vol. 47, no. 2, pp. 498-519, Feb. 2001.

[13] S. M. Kay, Fundamentals of Statistical Signal Processing, vol. I. Englewood Cliffs, NJ: Prentice-Hall, 1993.

[14] J. M. Mendel, Lessons in Estimation Theory for Signal Processing, Communications, and Control. Englewood Cliffs, NJ: Prentice-Hall, 1995.

[15] H. V. Poor and S. Verdú, "Probability of error in MMSE multiuser detection," IEEE Trans. Inf. Theory, vol. 43, no. 3, pp. 858-871, May 1997.

[16] E. Chiavaccini and G. M. Vitetta, "MAP symbol estimation on frequencyflat Rayleigh fading channels via a Bayesian EM algorithm," IEEE Trans. Commun., vol. 49, no. 11, pp. 1869-1872, Nov. 2001.

[17] W. Y. Zou and Y. Wu, "COFDM: An overview," IEEE Trans. Broadcast., vol. 41, no. 1, pp. 1-8, Mar. 1995.

[18] IEEE 802.11 TGn D2.00, Feb. 2007. [Online]. Available: http://www. ieee802.org/11/

[19] G. Caire, G. Taricoo, and E. Biglieri, "Bit-interleaved coded modulation," IEEE Trans. Inf. Theory, vol. 44, no. 3, pp. 927-946, May 1998.

[20] X. Li, H. C. Huang, G. J. Foschini, and R. A. Velenzuela, "Effects of iterative detection and decoding on the performance of BLAST," in Proc. IEEE Global Telecommun. Conf., 2000, vol. 2, pp. 1061-1066.

[21] L. B. Nelson and H. V. Poor, "Iterative multiuser receivers for CDMA channels: An EM-based approach," IEEE Trans. Commun., vol. 44, no. 12, pp. 1700-1710, Dec. 1996.

[22] S. Sun, T. T. Tjhung, and P. H. W. Fung, "Soft-decision based iterative interference cancellation (IIC) for group-wise STBC MIMO systems," in Proc. IEEE Veh. Technol. Conf.-Spring, Apr. 2003, vol. 2, pp. $984-988$

[23] M. Witzke, S. Bäro, F. Schreckenbach, and J. Hagenauer, "Iterative detection of MIMO signals with linear detectors," in Proc. 36th Asilomar Conf. Signals, Syst., Comput., Nov. 2002, vol. 1, pp. 289-293.

[24] X. Wang and H. V. Poor, "Iterative (turbo) soft interference cancellation and decoding for coded CDMA," IEEE Trans. Commun., vol. 47, no. 7, pp. 1046-1061, Jul. 1999.

[25] G. Begin and D. Haccoun, "High-rate punctured convolutional codes: Structure properties and construction technique," IEEE Trans. Commun., vol. 37, no. 12, pp. 1381-1385, Dec. 1989.

[26] G. Begin, D. Haccoun, and C. Paquin, "Further results on high-rate punctured convolutional codes for Viterbi and sequential decoding," IEEE Trans. Commun., vol. 38, no. 11, pp. 1922-1928, Nov. 1990. 
[27] Part 11: Wireless LAN Medium Access Control (MAC) and Physical Layer (PHY) Specifications: High-Speed Physical Layer in the $5 \mathrm{GHz}$ Band, Sep. 1999. IEEE Std. 802.11a-1999: Supplement to IEEE Std. 802.11-1999. IEEE 802

[28] C. Hermosilla and L. Szczeciński, "EXIT charts for turbo receivers in MIMO systems," in Proc. 7th ISSPA, Jul. 2003, pp. 209-212.

[29] S. ten Brink, "Convergence behavior of iteratively decoded parallel concatenated codes," IEEE Trans. Commun., vol. 49, no. 10, pp. 1727-1737, Oct. 2001.

[30] M. Tüchler and J. Hagenauer, "EXIT charts of irregular codes," in Proc. Conf. Inf. Sci. Syst., Mar. 2002, pp. 748-753.

[31] I. S. Reed, "On a moment theorem for complex Gaussian processes," IEEE Trans. Inf. Theory, vol. IT-8, no. 3, pp. 194-195, Apr. 1962

\section{A Modified Decorrelated Delay Lock Loop for Synchronous DS-CDMA Systems}

W. K. Wong, Y. T. Wu, S. H. Leung, and Y. S. Zhu

\begin{abstract}
This paper presents a modified decorrelated delay lock loop (MD-DLL) for synchronous direct-sequence code-division multiple-access (DS-CDMA) systems under multiuser environments. The structure of the MD-DLL is similar to that of a traditional delay lock loop (T-DLL), aside from the fact that the despreading signals in T-DLL are replaced by a linear combination of all the users' code sequences with various weightings and timing offsets. The basic principles of MD-DLL are given as follows: 1) to use the decorrelating technique of a multiuser detector to remove the multiple-access interference at the on-time code position instant and 2) to choose a proper timing offset for the code sequences to reduce the weighting imbalance between the two arms of the tracking loop and suppress noise enhancement. The proposed MD-DLL scheme solves the tracking bias problems in a decorrelated delay lock loop under a low-signal-to-noise-ratio (SNR) condition. Computer simulations based on Gold code sequences are used to verify the findings. It is shown that MD-DLL achieves a better and stable S-curve with negligible tracking bias, better rms tracking error and mean-time-to-lose-lock performance under high-SNR conditions, and stronger near-far resistance than T-DLL.
\end{abstract}

Index Terms-Delay lock loop, direct-sequence code-division multiple access (DS-CDMA), multiple-access interference (MAI), synchronization.

\section{INTRODUCTION}

The direct-sequence code-division multiple-access (DS-CDMA) technique is widely used in present-day communication systems for its well-known advantages, such as antijam and multipath rejection. An important issue for the successful operation of a DS-CDMA system is the code synchronization between the locally generated despreading sequence and the incoming sequence. The synchronization process consists of two stages: 1) code acquisition, which performs the coarse code phase alignment, and 2) code tracking, which maintains fine phase alignment. In this paper, we focus on the code tracking part.

Manuscript received April 11, 2007; revised September 3, 2007 and December 4, 2007. This work was supported by the City University of Hong Kong under Project 7001855-570. The review of this paper was coordinated by Dr. X. Wang.

W. K. Wong and S. H. Leung are with the Department of Electronic Engineering, City University of Hong Kong, Kowloon, Hong Kong (e-mail: eewkwong@cityu.edu.hk; eeeugshl@cityu.edu.hk).

Y. T. Wu is with the Department of Electronic Engineering, City University of Hong Kong, Kowloon, Hong Kong, and also with The Key Labs of Integrated Microsystems, Shenzhen Graduate School, Peking University, Shenzhen 518055, China (e-mail: yatingwu@ @ityu.edu.hk).

Y. S. Zhu is with The Key Labs of Integrated Microsystems, Shenzhen Graduate School, Peking University, Shenzhen 518055, China (e-mail: zhuys@ szpku.edu.cn).

Digital Object Identifier 10.1109/TVT.2008.915516

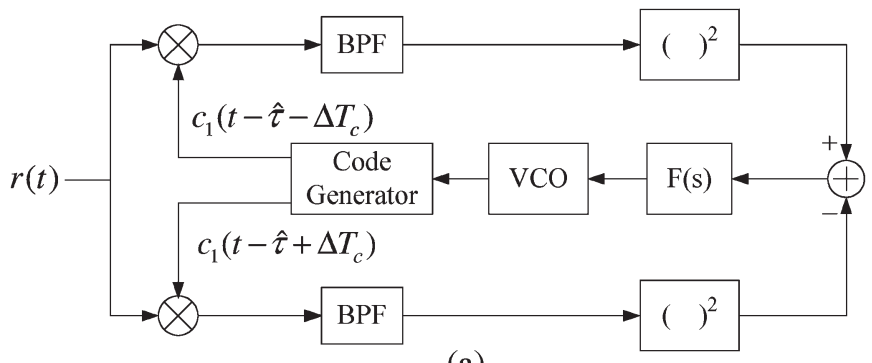

(a)

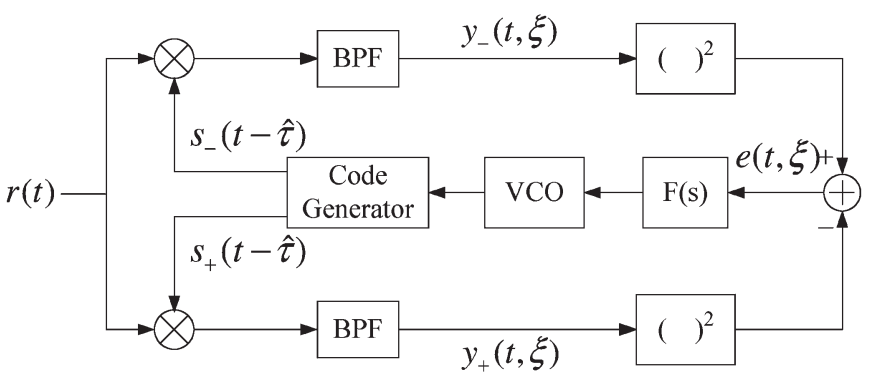

(b)

Fig. 1. Block diagram of (a) T-DLL and (b) MD-DLL.

The traditional delay lock loop (T-DLL) shown in Fig. 1(a) has been a well-proven technique for code tracking in DS-CDMA systems since its introduction in the 1960s [1]. T-DLL requires simple hardware implementation, and its performance is close to that of a maximumlikelihood estimator under an additive white Gaussian noise channel and single-user environment [2]. In a multiuser environment, the use of nonorthogonal code sequences causes multiple-access interference (MAI). Hong et al. [3] and Caffery and Stüber [4] have analyzed the effects of MAI on T-DLL with a Gaussian approximation for MAI and a simple approximation for the average aperiodic cross-correlation function between two sequences, respectively. Both have shown that the performance of T-DLL is highly degraded under strong MAI. It has also been demonstrated that the S-curve (i.e., the discriminator characteristic) is severely distorted, and the loop may become unstable for certain combinations of sequences [5], [6]. In addition, Wong [6] pointed out that a noticeable tracking bias may be created under imbalanced MAI. It has been shown that a 0.2 -chip tracking bias would cause noticeable degradation in most multiuser receivers [7], [8].

Interference cancellation techniques, such as Wang et al.'s successive interference cancellation [9] and Latva-aho and Lilleberg's parallel interference cancellation [10], have been applied to T-DLL to combat MAI. Both require accurate estimation before synchronization, and the hardware complexity increases with the number of interferers. A decorrelated delay lock loop (D-DLL) with simple hardware complexity has been proposed in [6]. It uses the decorrelating concept of the multiuser detector to solve the tracking bias and the stability problems under a high-signal-to-noise ratio (SNR) environment. However, a noticeable tracking bias may occur in D-DLL under low-SNR conditions. In this paper, we propose a modified D-DLL (MD-DLL) to combat the effects of MAI and achieve a negligible tracking bias over the SNR range of practical interest. The structure of MD-DLL is similar to that of T-DLL, aside from the fact that the despreading signals in T-DLL are replaced by a linear combination of weighted and shifted versions of all the users' code sequences. The hardware complexity is comparable to that of T-DLL and is independent of the number of interferers.

Here, we focus on the S-curve, rms tracking error, and mean-timeto-lose-lock (MTLL) performance of a first-order loop. The analysis 